\title{
Response to Forbes's comment
}

United European Gastroenterology Journal 2016, Vol. 4(1) 153

(C) Author(s) 2016

Reprints and permissions:

sagepub.co.uk/journalsPermissions.nav

DOI: $10.1177 / 2050640615614742$

\section{Carolina Ciacci ${ }^{1}$, Paul Ciclitira ${ }^{2,3}$, Marios Hadjivassiliou ${ }^{4}$, Katri Kaukinen ${ }^{5,6,7}$, Jonas F Ludvigsson ${ }^{8,9}$, Norma McGough ${ }^{10}$, David S Sanders ${ }^{11}$, Jeremy Woodward ${ }^{12}$, Jonathan N Leonard ${ }^{13}$ and Gillian L Swift ${ }^{14}$}

We disagree with the comment of Forbes regarding our misinterpretation of data from the study of Catassi et al. ${ }^{1}$ and our statement that a safe daily limit is probably in the region of $10 \mathrm{mg} / \mathrm{day}$.

First, in the Catassi et al. study the authors reported that 'some Celiac Disease patients showed a clear-cut worsening of the small-intestinal architecture after ingesting only $10 \mathrm{mg}$ gluten/day' and $1 / 13$ patients challenged with $10 \mathrm{mg}$ gluten/day developed a clinical relapse p.164.

Therefore, with the limit of the small number of patients challenged with $10 \mathrm{mg}$ gluten in this study $(n=13)$ some effects have been reported also for the quantity of $10 \mathrm{mg} /$ day.

The Food and Drug Administration (FDA) safety analysis ${ }^{2}$ concluded that $<1 \mathrm{ppm}$ of gluten in foods is required to protect the greatest number of patients. In the FDA report it is clearly specified that, in the gluten exposure studies examined, the results are not presented or interpreted within a toxicological risk assessment framework.

Therefore, for the FDA hazard/safety analysis, the results were reinterpreted within the frame of reference of $1 \mathrm{ppm}$.

The average no observable adverse effect level and the lowest observable adverse effect level associated with morphological effects were calculated utilising the appropriate uncertainty factor (that takes into account the variability among studies and data) and indicated as tolerable the acute intake level for gluten

\footnotetext{
${ }^{1}$ Department of Medicine and Surgery, University of Salerno, Salerno, Italy ${ }^{2}$ Department of Gastroenterology, Kings College London, London, UK

${ }^{3}$ The Rayne Institute, St Thomas Hospital, London, UK

${ }^{4}$ Academic Department of Neurosciences, Royal Hallamshire Hospital, Sheffield, UK

${ }^{5}$ School of Medicine, University of Tampere, Tampere, Finland

${ }^{6}$ Department of Internal Medicine, Tampere University Hospital, Tampere, Finland

${ }^{7}$ Department of Internal Medicine, Seinäjoki Central Hospital, Seinäjoki, Finland

${ }^{8}$ Department of Medical Epidemiology and Biostatistics, Karolinska Institutet, Stockholm, Sweden

${ }^{9}$ Department of Pediatrics, Örebro University Hospital, Orebro, Sweden
}

of $12.5 \mathrm{mg} / \mathrm{day}$, and for the subchronic exposure $0.4 \mathrm{mg}$ gluten/day and for chronic exposure $7.0 \mathrm{mg}$ gluten/day.

The FDA report, with the limitation of the available data, indicates as tolerable a quantity of gluten close to the one reported in our review. At the moment we do not have any reliable data for toxicity of gluten quantities in the range from $10-50 \mathrm{mg} /$ day. Therefore, on the basis of the scanty data we have, according to the FDA safety assessment analysis and with the limit of personal sensitivity, the only conclusion is that $50 \mathrm{mg}$ could be unsafe for most CD patients and $10 \mathrm{mg}$ should be safe for most of the patients.

Our final recommendation that the $\mathrm{CD}$ patients should be vigilant with their diet and avoid all sources of known gluten or contamination is the only reasonable conclusion.

\section{References}

1. Catassi C, Fabiani E and Iacono G. A prospective, double-blind, placebo-controlled trial to establish a safe gluten threshold for patients with celiac disease. Am J Clin Nutr 2007; 85: 160-166.

2. Office of Food Safety, Center of Food Safety and Applied Nutrition, Food and Drug Administration. Health hazard assessment for gluten exposure in individuals with celiac disease: Determination of tolerable daily intake levels and levels of concern for gluten, http://www.fda.gov/ downloads/Food/FoodScienceResearch/UCM264152.pdf (May 2011).

\footnotetext{
${ }^{10}$ Coeliac UK, High Wycombe, UK

${ }^{11}$ Gastroenterology and Liver Unit, Royal Hallamshire Hospital, Sheffield, UK

${ }^{12}$ Cambridge Intestinal Unit, Addenbrooke's Hospital, Cambridge, UK

${ }^{13}$ Department of Dermatology, St Mary's Hospital, London, UK

${ }^{14}$ Department of Gastroenterology, University Hospital Llandough, Cardiff, UK

Corresponding author:

Carolina Ciacci, Department of Medicine and Surgery, University of Salerno, Baronissi Campus, 84131 Salerno, Italy.

Email: cciacci@unisa.it
} 